\title{
Re: Confocal Laser Endomicroscopy in the Management of Endoscopically Treated Upper Urinary Tract Transitional Cell Carcinoma: Preliminary Data
}

\author{
Villa L1,2, Cloutier J1, Cotè JF3, Salonia A2,4, Montorsi F2,4, Traxer O1 \\ 1 Pierre and Marie Curie University Faculty of Medicine, Tenon Hospital, Department of Urology, Paris, France \\ 2San Raffaele Urological Research Institute, Unit of Urology, Division of Experimental Oncology, Milan, Italy \\ 3 Pierre and Marie Curie University Faculty of Medicine, Tenon Hospital, Department of Pathology, Paris, France \\ 4Vita-Salute San Raffaele University, Milan, Italy
}

J Endourol. 2016;30:237-242. doi: 10.1089/end.2015.0644. Epub 2015 Dec 30.

\section{EDITORIAL COMMENT}

We should accept that there are attempts in all over the world to do much less invasive treatments especially in surgical procedures. Nowadays, one of the interest areas of urologists is conservative management of upper urinary tract urothelial cancers (UUTCS). Is nephroureterectomy an overtreatment for all cases or which cases are eligible for minimally invasive therapy in UUTC. Recently, the European Association of Urology guidelines accepted conservative endoscopic treatment using ureterenoscopy for a selected group of UUTC patients with low-volume, low-grade, and low-stage disease. This study tried to explain a new technique, confocal laser endomicroscopy (CLE), for increasing accuracy of sampling in UUTC and improving the conservative management. CLE is an ultrahigh-resolution microscopy technique that allows imaging of tissue at a depth of $400 \mu \mathrm{m}$. A negative point for CLE is the sensitivity to tissue movement leading to motion artifacts that could result in blurred images. In other studies, the benefit of CLE was shown in colon, gastric and bronchial pathologies which are luminal organs like urinary tract. Eleven patients were enrolled in this study. Four patients were previously treated endoscopically and one patient had positive cytology with no evidence of a disease in the bladder. A digital ureteroscope Flex-XC was used in all cases. After fluorescein injection, real time video sequences were acquired. Biopsies were obtained from the suspicious lesions. According to CLE findings, there were three high-grade, seven low-grade tumors and one negative tumor. Biopsy findings revealed with four not reliable, one inflammation (negative one in CLE), one dysplasia, four low-grade tumors and one highgrade tumor. It is very hard to comment on a new technique with a small amount of patients. May be it is better to say the cliché words: This study should be confirmed or validated by wide variety of studies. In addition, although this study showed promises in the diagnosis and follow-up of UUTC with the support of studies on bladder cancer with CLE; $36 \%$ of all patients (four from all) had insufficient biopsy specimens. This means that new techniques and equipment should be developed to obtain reliable specimen by flexible endoscopes. It is probable that this evaluation will cause an increase in the applicability of this kind of techniques.

Barbaros Başeskioğlu, MD

\section{Re: The Functional Effects of Cigarette Smoking in Women on the Lower Urinary Tract}

\section{Madhu C, Enki D, Drake MJ, Hashim H}

Southmead Hospital and Bristol Urological Institute, Consultant Subspecialist Urogynecologist, Bristol, UK

Urol Int. 2015;95:478-482. doi: 10.1159/000438928. Epub 2015 Oct 10.

\section{EDITORIAL COMMENT}

Smoking is one of the major lifestyle risk factors affecting health. The role of smoking in triggering cancer in the lower urinary tract (LUT) is known, but its role in the pathogenesis of urinary incontinence needs to be investigated. In this study, the authors aimed to evaluate the urodynamic findings in female smokers with LUT symptoms. From a cohort of 11.678 women, who underwent urodynamic studies, $2.476(21.2 \%)$ reported to be a smoker. The mean age of the smokers and the non-smokers was 47 and 53.5 , respectively $(p<0.001)$. The rate of women reported to use anti-depressant in smoking group and non-smoker group was $13.6 \%$ and 8.4 , respectively $(\mathrm{p}<0.001)$. The proportion of menopausal women in smoking group was significantly less than in non-smoker group ( $50.5 \%$ vs. $62.8 \%, p<0.001$ ). Overactive bladder symptoms were more common in female smokers (Odds ratio $1.14, p=0.006$ ) and they had a slightly higher mean daytime frequency ( 8.54 vs. $7.92, p<0.001)$ but no nocturia episodes. A significant proportion of smoking women reported secondary nocturnal enuresis $(22.2 \%$ vs. $11.5 \%$, $\mathrm{p}<0.001)$ and coital incontinence. Smokers with LUT symptoms were more likely to have abnormal urodynamic abnormalities, such as detrusor overactivity (D0) and DO incontinence $(p<0.001)$. There was no difference in both groups' reports about stress urinary incontinence (SUI), but there was an increasing trend in the prevalence of SUI symptoms with increasing number of cigarettes. These findings may be because of nicotine and anti-estrogenic effects of smoking, affecting the collagen synthesis. Female smokers must be also questioned for LUT symptoms and, the importance of life style changes must be emphasized.

İlker Şen, MD 